\section{Groups and grandmothers in neuroscience}

SIR-In his review of Neural Darwinism: The Theory of Neuronal Group Selection (Nature 331, 571; 1988) Horace Barlow has made several factual errors and has misrepresented the theory by misinterpreting the nature of neuronal groups. Barlow's chief objection is that neuronal groups cannot carry out the functions proposed by the theory. This misinterpretation arises because he does not understand that the major mechanism of selection acts upon synapses in populations'. Barlow says that the theory uses Hebb's rule for synaptic plasticity. In fact, the book states explicitly (p.201) that these populations do not, in general, obey hebbian rules. This misunderstanding by Barlow invalidates all of his dependent arguments: in fact, increasing numbers of cells do not have to be incorporated into groups, experience makes the cells in a group more selective not less, and neuronal group selection confers a number of advantages that have been clearly outlined in the book. Not the least of these is that it accounts for the dynamic organization of topographic maps ${ }^{2}$.

Barlow is also confused about how receptive fields are represented by cells in groups. Receptive fields are similar within a group but not identical. Moreover, the purpose of cooperativity within a group is to provide sharp functional boundaries in an otherwise diverse and overlapping anatomy. The combinatorics afforded lead to an immense diversity: admittedly, the diversity is not as immense as would obtain from permuting individual neurons, but it is overwhelmingly larger than could be represented by Barlow's concept that individual neurons each "correspond[s] to a pattern of external events of the order of complexity of the events symbolized by a word"3 - the infamous 'grandmother' neuron.

Every reviewer has an inalienable right to sins of omission. This perhaps accounts for Barlow's overlooking the main themes of the book - the central importance of perceptual categorization, the constraints of development and evolution on brain function, the role of variability in the nervous system, and the various mechanisms of re-entry between maps. One can only suspect that his actual sins of commission in the review are motivated less by the presumption that the theory "ushers in a new era in neuroscience" than by the premonition that an old one is about to be ushered out.

The Rockefeller University, LEIF H. FinKel

New York, New York 10021, USA

1. Finkel, L.H. \& Edelman, G.M. Proc. natn. Acad. Sci. U.S.A. 82, 1291-1295(1985).

2. Pearson, J.C., Finkel, L.H. \& Edelman, G.M. J. Neurosci. 7, 4209-4223 (1987)

3. Barlow, H.P. Perception 1, 371-394 (1972)

BARLOW REPLIES-I found the book hard to understand, and the extravagant claims made for it perplexing, so I cannot dismiss the possibility that I failed to pick up some important messages. I am not convinced that this is so, but if it is I apologize and we must leave it to other, unbiased, readers to decide whether this was entirely my fault, or whether the book itself was to blame.

There are other bits of my review for which I do not apologize. Part of the book is concerned with background material which is unoriginal and adds no new insight, so I did not draw attention to it. I did mention the review of Edelman's own work on cell adhesion molecules, but it is disappointing that the work of other groups was not discussed. In the section on development there is no mention of work such as that of Willshaw, von der Malsberg or Swindale, which is certainly relevant to any discussion of the factors moulding the brain during development. It is perhaps unfair to expect an account of the spate of recent computer simulations of modifiable neural networks, but an uninformed reader might gain the impression that Edelman and his colleagues are the only ones with interests in this area, and that is unfortunate.

Finkel implies that I am wedded to, or even responsible for, the notion of 'grandmother' neurons. If he would re-read the
SIR-As Stewart et al.' have pointed out, there are theoretical reasons to expect the evolution of protein sequences to be divergent, and there exists abundant experimental evidence from many kinds of protein to suggest that it is. The theoretical argument applies with particular force to the bacteriolytic function of lysozyme, which can be imitated with random copolymers of glutamate and phenylalanine ${ }^{2.3}$. As there are many more than twenty families of proteins for which at least six sequences are available for comparison, one ought to be surprised if one of them did not display characteristics with a likelihood less than $5 \%$, and so to be convinced of the reality of convergence in the evolution of sequences one can hardly be satisfied with a result significant in a $95 \%$ confidence test.

The sequences of lysozyme from langur, baboon, human, rat, cattle and horse contain four loci at which the langur and cattle enzymes share identical residues not found in any other vertebrate lysozymes apart from other ruminant or colobine stomach enzymes, whereas other pairs from the six sequences share either one such identity or none'. As the seven identities could in principle be distributed

article he quotes he would find that its ideas are less restrictive than he thinks: the cells postulated are thought to owe their properties partly to modification by experience, and room is left for cooperative or connectionist factors to mould the neurons that categorize perceptions. Room is also left for neurons that are selectively sensitive to highly specific stimuli, such as the faces of particular individuals. Finkel has a right to regard such neurons as "infamous", but I hope this does not blind him to the experimental evidence of Gross, Rolls, Perrett and their colleagues ${ }^{1,3}$ that they exist.

Although I do not agree with the ideas in Neural Darwinism I think the title is splendid, and it should encourage all of us to seek a role for selection in neurobiology. Experimental techniques are developing rapidly, and we need as many clearly expressed theories as possible about the way the brain performs its remarkable functions; the facts elicited by testing them will then weed out errors and select the fittest ideas with greater finality than any amount of discussion in these columns.

Horace Barlow

The Physiological Laboratory,

University of Cambridge,

Cambridge CB2 3EG, UK

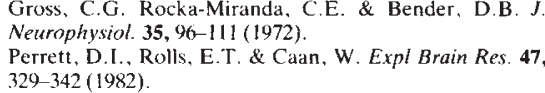

Perrett, D I., Rolls, E.T. \& Caan, W. Expl Brain Res. 47, 329-342 (1982).

3. Perrett, D.I. et al. Hum. Neurobiol. 3, 197-208(1984).

\title{
Convergent evolution of lysozyme sequences?
}

at random among 15 pairs of sequences, one can readily calculate the probability that four or more would occur by chance in the same pair of sequences as $15 p^{4}\left(p^{3}+\right.$ $\left.7 p^{2} q+21 p q^{2}+35 q^{3}\right)=0.0088$, where $p=$ $1 / 15$ and $q=14 / 15$. This is smaller than implied by the reported significance in a $95 \%$ test', but is too large to exclude the possibility that one is dealing with more than the expected tail of the distribution.

When the lysozyme sequences were analysed on the assumption that the true phylogenetic tree linking them is the ordinary biological tree in which the langur is more closely related to the baboon than to cattle, five amino-acid substitutions were placed on the lineage leading to langur from the ancestor unique to langur and baboon. The probability of observing as many as five on this lineage was also assessed as less than $5 \%$. But the particular placing of substitutions represents an interpretation of the observations, not the observations as such, and the putative substitutions cannot be analysed statistically as if they were actually observed. At locus 50, for example, the occurrence of glutamate in cattle and langur but glutamine in the rat and baboon was interpreted as two 